\title{
含偶氮苯侧链分子刷聚合物的合成及其光响应自组装
}

\author{
钱鸿宇何品张璐陈珂徐涁涁林绍梁* \\ (华东理工大学材料科学与工程学院 上海 200237)
}

\begin{abstract}
摘要 分子刷聚合物伸展的高分子主链、高密度的侧链和较低的侧链空间缠结, 使其展现出独特的流变学、力学性能 和特殊的分子聚集状态，在纳米技术、表面科学等领域有着广泛的应用前景. 基于同时含有 2-羰基溴与炔基基团的大 分子试剂聚 2-((2-溴代丙酰氧基)甲基)丙烯酸丙炔酯, 引发偶氮苯丙烯酸酯单体的原子转移自由基聚合(ATRP)与叠氮 功能化的巴比妥酸衍生物的点击反应, 合成了新型含偶氮苯侧链的分子刷聚合物聚丙烯酸酯- $g$-聚(6-(4-丁基-4'-氧偶氮 苯)正己基丙烯酸酯)/巴比妥酸(PA-g-PAzo/Bar), 研究了其在溶液中的自组装和光响应性行为. 随着分子刷聚合物浓度 的增加, 聚集体由柱状胶束向复合胶束转变. 由于光响应性偶氮苯侧链的存在, 在紫外光的照射下偶氮苯生色团发生 trans-cis 异构化转变, 促使柱状胶束融合形成多孔网状聚集体, 同时球形复合胶束融合形成珍珠项链状聚集体.
\end{abstract}

关键词 偶氮苯; 分子刷聚合物; 光响应性; 超分子自组装

\section{Synthesis and Photo-responsive Self-Assembly of Azobenzene-Containing Molecular Brushes}

\author{
Qian, Hongyu $\quad \mathrm{He}$, Pin $\quad$ Zhang, Lu Chen, Ke $\quad \mathrm{Xu}$, Binbin Lin, Shaoliang*
}

(School of Materials Science and Engineering, East China University of Science and Technology, Shanghai 200237)

\begin{abstract}
Molecular brushes (MBs) are a unique type of branched macromolecules which graft polymeric chains onto a linear polymeric backbone densely. Crowded tethered side chains generate strong steric repulsion and force the backbone to stretch out, resulting in a persistent cylindrical shape and low density of entanglements of brushes. Owing to the unique rheological and mechanical properties and self-assembly behaviors, MBs show a great potential in nanotechnology and surface science. Herein, the synthesis of novel MBs of polyacrylate-g-poly(6-(4-butyl-4'-oxyazobenzene)hexylacrylate)/barbituric acid (PA-g-PAzo/Bar) was reported, in which PAzo brushes and hydrogen-bonding barbituric acid were grafted using the combination of concurrent atom transfer radical polymerization (ATRP) and click reaction. MBs-based aggregates change from cylinder to compound micelle by increasing the concentration. It is interesting that morphology transitions of cylinder-to-porous nanosheet and compound micelle-to-pearl-necklace were observed upon UV irradiation, that was ascribed to the trans-to-cis isomerization of PAzo brushes.
\end{abstract}

Keywords azobenzene; molecular brushes; photo-responsiveness; supramolecular self-assembly

嵌段共聚物(BCP)的自组装行为可以简单高效地构 筑微纳米精细结构, 受到了高分子科学家的广泛关 注 ${ }^{[1-4]}$. 通过调节嵌段共聚物中不同链段的嵌段比，可以 制备一系列形貌丰富多样的聚集体 ${ }^{[5-7]}$. 伴随着活性/可 控自由基聚合和点击化学等的出现，一系列模仿自然界 复杂且精细结构的高分子聚合物被设计合成，包括星 状、环状、接枝和树状聚合物等 ${ }^{[8-10]}$. 这些不同结构聚 合物的出现不仅为探究聚合物结构与性能之间的关系 提供了便利, 也大大拓宽了聚合物的实际应用价
值 ${ }^{[11-13]}$. 众多结构复杂的聚合物中，分子刷聚合物 (Molecular Brushes) 是极为特殊的一种, 由聚合物侧链 高密度地接入至另一聚合物主链中, 形成主链与支链相 连接的刷状结构, 兼具主链和支链的性能 ${ }^{[14-16]}$. 分子刷 聚合物具有高密度的侧链结构、伸展的高分子主链以及 较低的侧链空间缠结, 展现出很多嵌段共聚物所不具备 的性质, 如独特的流变学与力学性能、特殊的单分子聚 集状态、本体相分离行为等, 在纳米技术、生物医学、 表面科学、超分子科学和聚合物-无机纳米复合材料等

\footnotetext{
* Corresponding author. E-mail: slin@ecust.edu.cn

Received February 25, 2021; revised March 25, 2021; published online April 29, 2021.

Project supported by the National Natural Science Foundation of China (Nos. 52073092, 51873061, 22001072).

国家自然科学基金(Nos. 52073092, 51873061, 22001072)资助项目.
} 
领域有着广泛的应用前景 ${ }^{[17-18]}$. 然而, 分子刷聚合物的 自组装行为还较少被报道, 特别是将不同功能性的侧链 同时引入到分子刷聚合物中, 构建功能化的分子刷聚合 物组装体还有待研究, 这也限制了分子刷聚合物进一步 的应用.

偶氮苯衍生物作为一类重要的液晶与光响应材料, 具有良好的加工性、成膜性、快速响应性和取向固定性, 在光机械相转变、光控可逆性能转变和光控纳米材料等 领域存在巨大的应用前景 ${ }^{[19-20]}$. 偶氮苯材料的光响应性 主要体现于其光致顺反异构化的特性, 处于基态的反式 结构呈现平面棒状形态, 具有较强的 $\pi-\pi$ 共轭作用, 聚 集态表现出液晶行为; 处于亚稳态的顺式结构为拐式形 状, 无液晶行为, 具有一定的亲水性. 近年来, 制备含 有偶氮苯生色团的均聚物和共聚物, 研究其光响应性自 组装行为受到了广泛的关注, 并构建了一系列智能的光 响应材料 ${ }^{[21-22]}$. 将偶氮苯引入到分子刷聚合物中, 不仅 可以赋予组装体对光的刺激响应性, 还可以拓展其实际 应用领域 ${ }^{[23]}$.

本文采用同时含有 2-羰基溴与炔基基团的大分子 试剂聚 2-((2-溴代丙酰氧基)甲基)丙烯酸丙炔酯

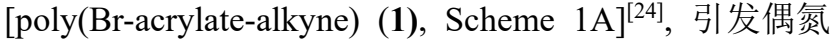
苯丙烯酸酯单体 2 的原子转移自由基聚合(ATRP)与叠 氮功能化的巴比妥酸衍生物 $\mathbf{3}$ 的点击反应，合成了新型 含偶氮苯侧链的分子刷聚合物聚丙烯酸酯- $g$-聚(6-(4-丁 基-4'-氧偶氮苯)正己基丙烯酸酯)/巴比妥酸[PA- $g$-PAzo/ Bar (4), Scheme 1B]. 其中偶氮苯侧链具有光响应性以 及较强的 $\pi-\pi$ 共轭作用, 可以赋予组装体规整的微观排 布和光诱导的形貌转变能力; 巴比妥酸链段可以提供强 氢键作用，促使组装体形成特殊的形态结构，并能够长 时间稳定存在 ${ }^{[25-26]}$. 我们详细研究了分子刷聚合物 PA-g-PAzo/Bar 在溶液中的自组装和光响应性行为. 随 着分子刷浓度的增长, PA-g-PAzo/Bar 聚集体由柱状胶 束向复合胶束转变. 由于光响应性偶氮苯侧链的存在, 在紫外光的照射下偶氮苯生色团发生 trans-cis 异构化的 转变, 促使柱状胶束融合形成多孔网状聚集体，同时球 形复合胶束融合形成珍珠项链状聚集体.

\section{1 结果与讨论}

\section{1 含偶氮苯侧链分子刷聚合物 PA-g-PAzo/Bar 合成} 含偶氮苯侧链分子刷聚合物 PA-g-PAzo/Bar (4)的合

(A)
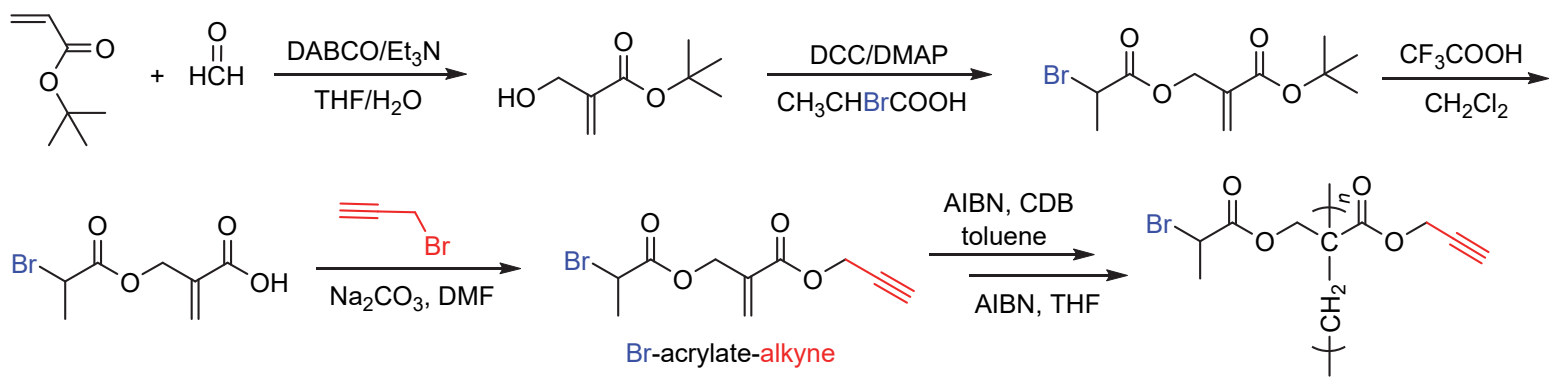

poly(Br-acrylate-alkyne) (1)

(B)
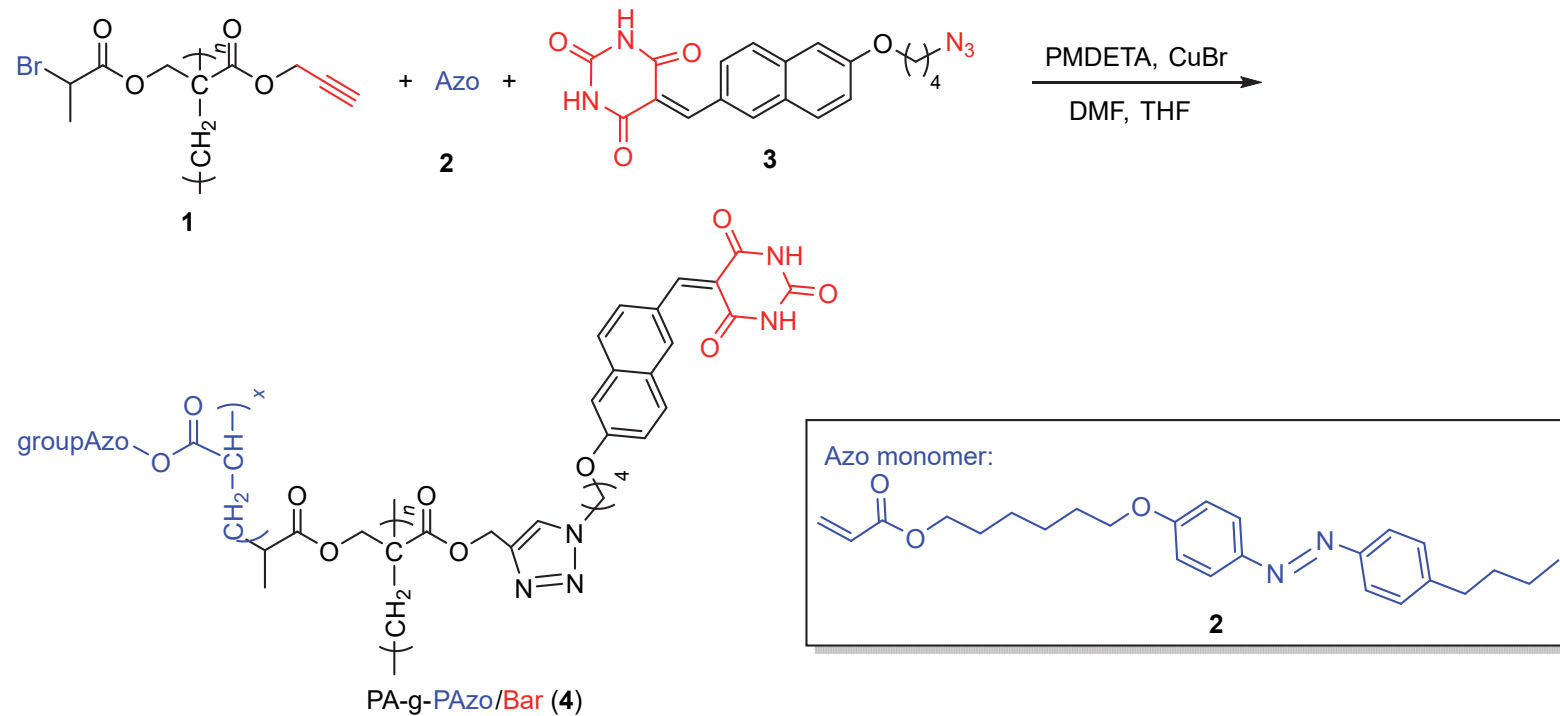

图式 1 (A) poly(Br-acrylate-alkyne) (1)和(B) PA-g-PAzo/Bar (4)的合成

Scheme 1 (A) Syntheses of poly(Br-acrylate-alkyne) (1), and (B) PA-g-PAzo/Bar (4) 
成如 Scheme 1 所示: 在 $N, N$-二甲基甲酰胺(DMF)和四 氢呋喃(THF)的混合溶剂中, 以同时含有 2-羰基溴与炔 基基团的 poly(Br-acrylate-alkyne) (1)为大分子试剂, 五 甲基二亚乙基三胺(PMDETA)为配体, 澳化亚铜 $(\mathrm{CuBr})$ 为催化剂, 引发偶氮苯丙烯酸酯单体 $\mathbf{2}$ 的 ATRP 与叠氮 功能化的巴比妥酸衍生物 $\mathbf{3}$ 的点击反应, 制备得到分子 刷聚合物 $\mathrm{PA}_{28}-g-\mathrm{PAzO}_{17} / \mathrm{Bar}$ (下标为从 ${ }^{1} \mathrm{H}$ NMR 计算得出 的聚合度). 其中, 大分子试剂 poly(Br-acrylate-alkyne) 和偶氮苯丙烯酸酯单体根据课题组以往的报道设计合 成 ${ }^{[24]}$. 叠氮功能化的巴比妥酸衍生物根据文献报道合 成 ${ }^{[27-28]}$ : 6-差基-2-荎甲酫与 1,4-二溴丁烷反应制备得到 化合物 S1, 随后与叠氮化钠反应得到叠氮功能化的化 合物 $\mathbf{S 2}$, 化合物 $\mathbf{S 2}$ 与巴比妥酸反应合成得到叠氮功能 化的巴比妥酸衍生物 $\mathbf{S 3}$.

运用核磁共振氢谱 $\left({ }^{1} \mathrm{H}\right.$ NMR) 和凝胶渗透色谱 (GPC) 对聚合物的结构、分子量进行了表征. 分子刷聚 合物 PA- $g$-PAzo/Bar 的 ${ }^{1} \mathrm{H}$ NMR 如图 1A 所示, $\delta 6.80 \sim$ 8.00 处的质子峰是偶氮苯侧链的特征信号, 说明通过 ATRP 成功引入了偶氮苯侧链; $\delta 8.27$ 和 10.08 处的质子 峰分别是巴比妥酸连接的双键和酰胺的特征信号, 说明 通过点击反应成功地引入了巴比妥酸结构. 如图 $1 \mathrm{~B}$ 所 示, 分子刷聚合物 PA-g-PAzo/Bar 的 GPC 曲线呈对称的 单峰, 聚合物分子量为 $M_{\mathrm{n}}=30100$, 分子量分布 $(\theta)$ 为 1.27 , 说明聚合反应的成功进行, 分子间偶合反应可以 被忽略.

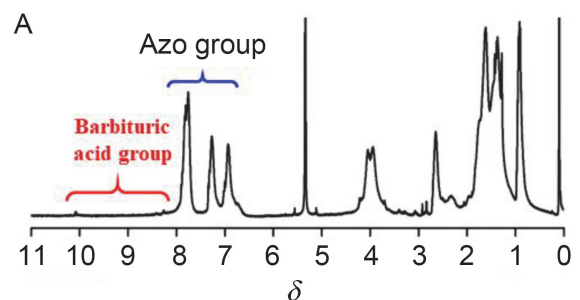

B

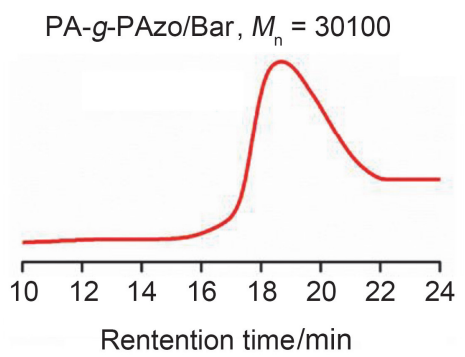

图 1 分子刷聚合物 PA-g-PAzo/Bar (4)的(A)核磁共振氢谱和 (B) GPC 谱图

Figure 1 (A) ${ }^{1} \mathrm{H}$ NMR spectrum and (B) GPC trace of PA-g-PAzo/Bar (4) of PA-g-PAzo/Bar (4)

\section{2 分子刷聚合物 PA- $g$-PAzo/Bar 胶束的制备与光致 异构化行为}

将分子刷聚合物 PA- $g$-PAzo/Bar 溶于氯仿中, 随后
向其缓慢滴加选择性溶剂甲基环已烷 $(\mathrm{MCH})$ ，制备了浓 度为 $0.2 \mathrm{mg} \cdot \mathrm{mL}^{-1}$ 的聚合物胶束溶液, 具体制备过程见 实验部分. 通过紫外-可见(UV-vis)吸收光谱研究 PA-g$\mathrm{PAzo} / \mathrm{Bar}$ 胶束溶液的光致异构化行为. 图 $2 \mathrm{~A}$ 为波长 $365 \mathrm{~nm}$ 的紫外光照射不同时间后, PA-g-PAzo/Bar 胶束 溶液的紫外-可见吸收光谱，对应偶氮苯生色团电子的 $\pi \rightarrow \pi^{*}$ 跃迁的反式结构吸收峰位于 $350 \mathrm{~nm}$ 处. 当使用波 长 $365 \mathrm{~nm}$ 的紫外光照射时, $350 \mathrm{~nm}$ 处反式偶氮苯的吸 收峰随光照时间的延长逐渐减弱, $445 \mathrm{~nm}$ 处顺式偶氮苯 的吸收峰随光照时间的延长逐渐增强. 照射 $30 \mathrm{~s}$ 后体系 达到稳定状态，此时偶氮苯 trans-和 cis-构型转变达到 平衡. 图 2B 为该胶束溶液在 $450 \mathrm{~nm}$ 蓝光照射下回复过 程的 UV-vis 谱图，经过 $20 \mathrm{~s}$ 照射后, cis-构型重新回复为 原先较为稳定的 trans-构型, 伴随着 $350 \mathrm{~nm}$ 处反式偶氮 苯吸收峰的逐渐增强, $445 \mathrm{~nm}$ 处顺式偶氮苯吸收峰逐渐 减弱. PA-g-PAzo/Bar 在 $365 \mathrm{~nm}$ 紫外光和 $450 \mathrm{~nm}$ 蓝光下
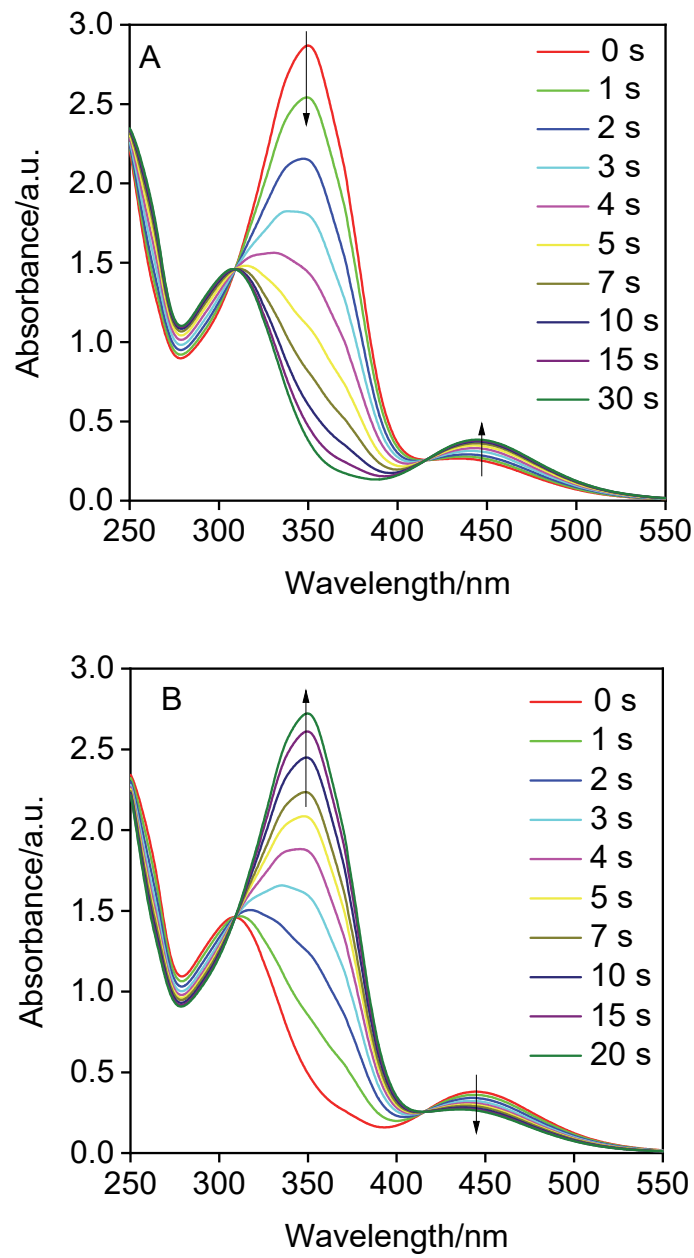

图 2 分子刷聚合物 $\mathrm{PA}-\mathrm{g}-\mathrm{PAzo} / \mathrm{Bar}$ 胶束溶液 $\left(0.2 \mathrm{mg} \cdot \mathrm{mL}^{-1}\right.$, $\left.\mathrm{CHCl}_{3} / \mathrm{MCH}, V: V=1: 0.2\right)$ 的紫外吸收光谱图

Figure 2 UV-Vis absorption spectra of PA-g-PAzo/Bar micelle solution $\left(0.2 \mathrm{mg} \cdot \mathrm{mL}^{-1}, \mathrm{CHCl}_{3} / \mathrm{MCH}, V: V=1: 0.2\right)$

(A) Upon irradiation of $365 \mathrm{~nm}$ light; (B) upon irradiation of $450 \mathrm{~nm}$ light 
的光致异构化构型转变可以连续重复, 吸收峰基本能够 回复到最初的强度, 表明聚合物胶束溶液的光致异构化 过程较为稳定.

\section{3 分子刷聚合物 PA-g-PAzo/Bar 的溶液自组装行为}

按照实验部分胶束的制备步骤，在氯仿和甲基环己 烷的混合溶剂(体积比为 $1: 0.2$ )中, 制备了浓度分别为 0.2 与 $1 \mathrm{mg} \cdot \mathrm{mL}^{-1}$ 的聚合物胶束溶液, 其中氯仿是聚合 物的良溶剂, 而甲基环已烷对分子刷的主链和偶氮苯侧 链有一定的溶解性, 对巴比妥酸链段是不良溶剂, 因此 作为聚合物的选择性溶剂. 研究了 PA-g-PAzo/Bar 分子 刷聚合物浓度对组装体形貌的影响, 形貌随聚合物浓度 变化的典型透射电子显微镜(TEM)图像如图 3 所示. 当 浓度为 $0.2 \mathrm{mg} \cdot \mathrm{mL}^{-1}$, 组装体形貌为短柱状 (图 3A, 3B), 长度与直径分别为 $50,15 \mathrm{~nm}$ 左右. 这些柱状胶束是由 偶氮苯侧链为壳, 巴比妥酸部分为核所组成, 其中巴比 妥酸可以在胶束内部形成强的氢键作用, 使得胶束更为 稳定地存在. 由于分子刷聚合物特殊的柱状分子构象以 及偶氮苯侧链的 $\pi-\pi$ 共轭作用, 赋予了聚集体较高的末 端活性 ${ }^{[29]}$. 因此, 发现所制备的柱状胶束随着储存时间 的延长, 在偶氮苯侧链 $\pi-\pi$ 共轭作用的促进下, 活性的 末端与末端逐渐相互连接, 形成了更长的柱状胶束(图 3C). 如图 3D 所示, 进一步提高聚合物的浓度, 当浓度 为 $1 \mathrm{mg} \cdot \mathrm{mL}^{-1}$ 时, 组装体为球形复合胶束, 尺寸并不均 一, 直径在 $80 \sim 150 \mathrm{~nm}$ 之间. 这些球形复合胶束的内部 由许多小胶束组成, 因此直径远远大于聚合物链伸展的 长度. PA-g-PAzo/Bar 分子刷聚合物所形成的柱状胶束 与复合胶束的结构示意图如图 3E 所示, 红色部分代表 疏溶剂的巴比妥酸链段, 蓝色部分代表较为亲溶剂的聚 合物主链与偶氮苯侧链.

\section{4 分子刷聚合物 PA-g-PAzo/Bar 胶束的光诱导形貌 转变}

由于 PA-g-PAzo/Bar 中的偶氮苯侧链具有光响应特 性, 在紫外光的照射下, 偶氮苯生色团由反式构型向顺 式构型转变, 非平面结构的顺式偶氮苯的比例大大增 加. 这种转变会导致含偶氮苯链段的聚集体的极性增 加, 同时破坏了偶氮苯链段在聚集体中的排列并引起缺 陷, 为了降低体系自由能和稳定聚集体, 聚集体会发生 形貌的转变, 从而赋予聚集体形貌的光响应性. 如图 4 所示, 在紫外光的照射下, 柱状胶束转变形成多孔网状 聚集体(图 4A). 推测偶氮苯侧链顺反异构的变化, 导致 初始的短柱状胶束处于亚稳状态, 但是由于巴比妥酸强 的氢键交联作用, 胶束并未解离, 而是逐渐融合、堆积, 形成多孔纳米板结构, 如图 4C 所示. 多孔网状结构的 放大的 TEM 图像如图 4B 所示, 进一步说明网状聚集体 是由许多短柱状胶束组成.
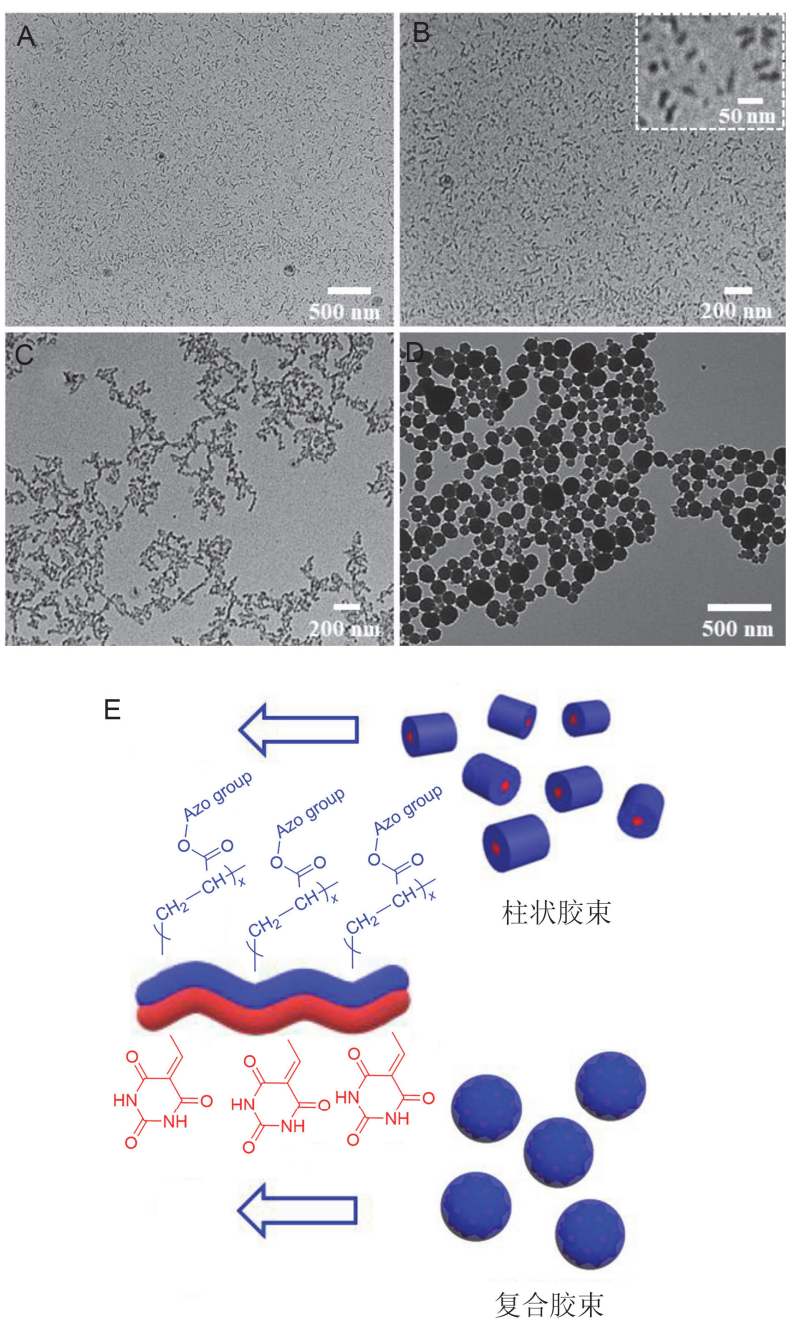

图 3 分子刷聚合物 PA- $g$-PAzo/Bar 胶束溶液(A, B) $0.2 \mathrm{mg}$ • $\mathrm{mL}^{-1}$ 样品、(C) $0.2 \mathrm{mg} \cdot \mathrm{mL}^{-1}$ 样品存储 $7 \mathrm{~d}$ 后和(D) $1 \mathrm{mg} \cdot \mathrm{mL}^{-1}$ 样品的 TEM 图像; (E)制备所得的柱状胶束和复合胶束的示意 图

Figure 3 TEM images of PA- $g$-PAzo/Bar micelle solution: (A, B) $0.2 \mathrm{mg} \cdot \mathrm{mL}^{-1}$ micelle solution, (C) $0.2 \mathrm{mg} \cdot \mathrm{mL}^{-1}$ micelle solution after storing $7 \mathrm{~d}$, and (D) $1 \mathrm{mg} \cdot \mathrm{mL}^{-1}$ micelle solution; (E) Schematic illustration of the obtained cylinder and compound micelles

随后，研究了分子刷聚合物 PA-g-PAzo/Bar 所形成 的球形复合胶束在紫外光照后形貌的变化. 同样, 在紫 外光的照射下, 非平面结构的顺式偶氮苯的比例大大增 加，复合胶束壳层中偶氮苯链段的稳定排列被破坏，导 致复合胶束外部处于亚稳状态，而巴比妥酸部分的交联 作用可以保证整个胶束不被破坏. 因此, 发现在紫外光 照 $1 \mathrm{~h}$ 后球形复合胶束出现了一定程度的连接与融合, 形成短链结构, 如图 5A 所示. 图 5B 是短链结构放大的 TEM 图像, 可以清晰地发现短链结构的内部由多个复 合胶束相互连接组成. 进一步延长紫外光照时间至 $3 \mathrm{~h}$, 如图 5C 所示, 球形复合胶束基本完全连接与融合, 形 成珍珠项链状结构. 图 5D 是珍珠项链状结构的放大的 


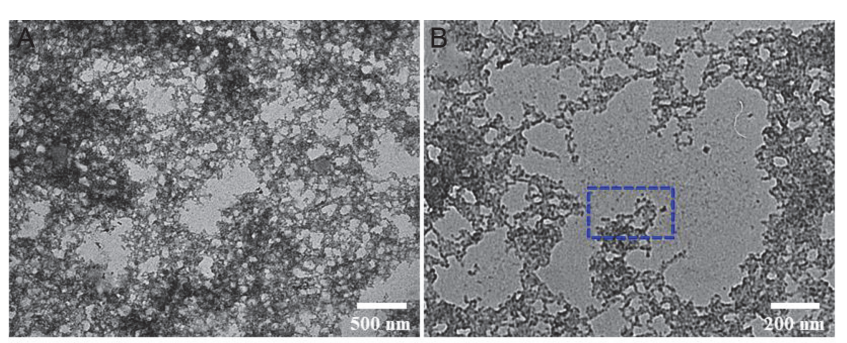

c

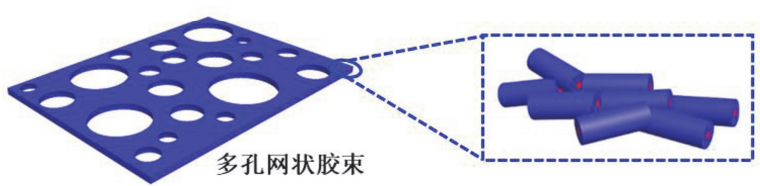

图 4 (A), (B)分子刷聚合物柱状胶束经过紫外光照后的 TEM 形貌图像. (C)柱状胶束经过紫外光照后形成多孔网状胶束的 示意图

Figure 4 (A), (B) TEM images of PA-g-PAzo/Bar cylinder micelles after UV irradiation. (C) Simplified schematic illustration of the obtained porous nanosheet after UV irradiation

TEM 图像，进一步证明球形复合胶束之间的紧密融合. 图 5 给出了在紫外光照下, 聚集体由球形复合胶束到短 链胶束, 最后转变为珍珠项链状胶束的示意图.

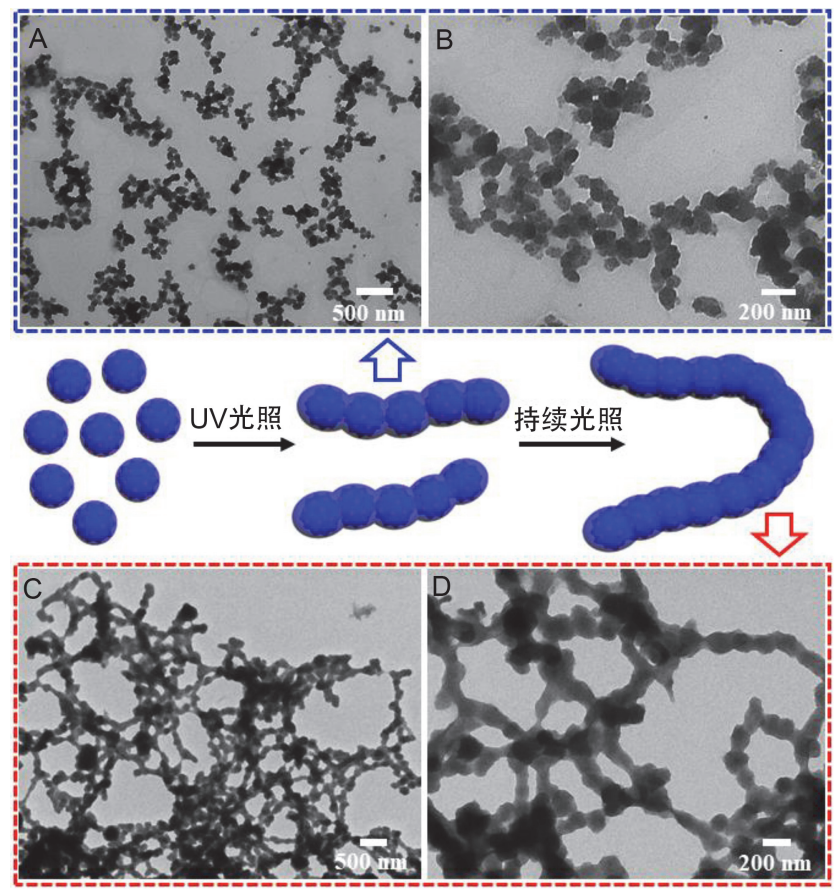

图 5 分子刷聚合物 PA- $g-P A z o / B a r$ 复合胶束经过紫外光(A, B)照射 $1 \mathrm{~h}$ 和(C, D) $3 \mathrm{~h}$ 后的 TEM 形貌图

Figure 5 TEM images of PA- $g$-PAzo/Bar compound micelles after UV irradiation (A, B) $1 \mathrm{~h}$, and (C, D) $3 \mathrm{~h}$

\section{2 结论}

基于含有 2-羰基溴与炔基基团的大分子试剂 poly(Br-acrylate-alkyne), 通过 ATRP 和点击反应, 一锅法合 成了同时含偶氮苯侧链和巴比妥酸链段的分子刷聚合
物 PA-g-PAzo/Bar. 结合光响应性的偶氮苯侧链和强氢 键作用的巴比妥酸链段, 分子刷聚合物在溶液中显示出 有趣的光响应性自组装行为. 随着分子刷聚合物浓度的 增加, PA- $g$-PAzo/Bar 聚集体由柱状胶束向复合胶束转 变. 在紫外光的照射下, 偶氮苯生色团发生 trans-cis 异 构化的转变, 促使柱状胶束融合形成多孔网状聚集体, 同时球形复合胶束融合形成珍珠项链状聚集体. 分子刷 聚合物 PA-g-PAzo/Bar 的成功合成, 不仅丰富了偶氮苯 聚合物的结构与类型，也为制备智能响应性的功能纳米 材料提供了新思路.

\section{3 实验部分}

\section{1 仪器与试剂}

6-羟基-2-菜甲醛(98\%，TCI)、1,4-二溴丁烷 $(98 \%$, TCI)、巴比妥酸( $98 \%, \mathrm{TCI}) 、$ 溴化亚铜(99\%, Aldrich); 五 甲基二乙烯三胺(PMDETA，99\%，Aldrich); 四氢呋喃 (THF, AR, Adamas); $N, N$-二甲基甲酰胺(DMF, AR, Adamas); 正已烷(AR, Adamas); 乙酸乙酯(EA, AR, Adamas); 透射电镜铜网: 北京新兴百瑞技术有限公司, $\mathrm{T} 10024$ 碳支持膜; 透析袋: 截留分子量 $3500 \mathrm{Da}$ ，上海 绿鸟科技发展有限公司; 实验用水为超纯水, 自制.

核磁共振分析仪(Advance 400, 德国 Bruker 公司)、 傅立叶变换红外光谱仪(Nicolet-170X, 美国热电公司)、 紫外分光光度计(UV-2550, SHIMADZU 公司)、透射电 子显微镜(TEM, JEM-1400 型, JEOL 公司)、紫外 LED 灯(UP114 型, Uvata 公司)、凝胶色谱仪(Waters 1515, Waters 公司). 采用 Waters 2410 示差折光检测器和 Waters 2487 紫外检测器, 用 THF 将聚合物配成 1 $\mathrm{mg} \cdot \mathrm{mL}^{-1}$ 的溶液, 淋洗液为 THF, 流速 $1 \mathrm{~mL} \cdot \mathrm{min}^{-1}$, 采用线性聚甲基丙烯酸甲酯(PMMA)标样对分子量 进行校正.

\section{2 实验方法}

\subsubsection{6-(4-澳丁氧基)-2-菜甲醛 $(\mathbf{S 1})$ 的合成}

氮气氛围下, 向预置磁子的 $100 \mathrm{~mL}$ 干燥三颈瓶中 加入 $\mathrm{K}_{2} \mathrm{CO}_{3}(2.7 \mathrm{~g}, 19.5 \mathrm{mmol}), 1,4$-二溴丁烷 $(4.2 \mathrm{~g}, 19.5$ $\mathrm{mmol}$ )和干燥的 DMF $(20 \mathrm{~mL})$. 将 6-羟基-2-芸甲醛 (2.2 $\mathrm{g}, 13.0 \mathrm{mmol}$ )溶于 $10 \mathrm{~mL}$ DMF，缓慢滴加到三颈瓶溶液 中, 室温摚拌 $2 \mathrm{~h}$, 升至 $70{ }^{\circ} \mathrm{C}$ 继续摚拌 $2.5 \mathrm{~h}$. 将反应液 抽滤后, 用饱和食盐水洗 3 次 $(50 \mathrm{~mL} \times 3)$, 乙酸乙酯萃 取 $(50 \mathrm{~mL} \times 3)$, 过硅胶柱(淋洗剂: 正己烷/乙酸乙酯, $V: \quad V=20 ： 1$ )得到 $2.6 \mathrm{~g} \mathrm{6}$-(4-溴丁氧基)-2-芸甲酫 (S1) $)^{[26,30]}$, 产率 65\%. m.p. 85 86 ${ }^{\circ} \mathrm{C} ;{ }^{1} \mathrm{H}$ NMR $(400$ $\left.\mathrm{MHz}, \mathrm{CD}_{2} \mathrm{Cl}_{2}\right) \delta: 10.09(\mathrm{~s}, 1 \mathrm{H}), 8.29(\mathrm{~s}, 1 \mathrm{H}), 7.90(\mathrm{~m}, 3 \mathrm{H})$, 7.25 (dt, $J=5.6,2.4 \mathrm{~Hz}, 2 \mathrm{H}), 4.18(\mathrm{t}, J=6.0 \mathrm{~Hz}, 2 \mathrm{H}), 3.56$ (t, $J=6.5 \mathrm{~Hz}, 2 \mathrm{H}), 2.02 \sim 2.20(\mathrm{~m}, 4 \mathrm{H})$. 


\section{$3.2 .26-(4-$ 叠氮丁氧基)-2-䒬甲醛(S2)的合成}

向预置磁子的 $250 \mathrm{~mL}$ 干燥三颈瓶中, 加入 $\mathrm{NaN}_{3}$ (3.3 g, $50 \mathrm{mmol})$, 化合物 $\mathbf{S 1}(2.5 \mathrm{~g}, 8.2 \mathrm{mmol})$ 和干燥的 DMF $(120 \mathrm{~mL})$, 升温至 $50{ }^{\circ} \mathrm{C}$, 反应 $48 \mathrm{~h}$. 停止加热并 冷却至室温, 过滤除去过量的叠氮化钠. 加入过量饱和 食盐水 $(300 \mathrm{~mL})$, 乙酸乙酯萃取 5 次 $(100 \mathrm{~mL} \times 5)$, 经无

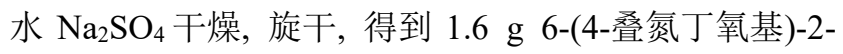
菜甲醛 $(\mathrm{S} 2)^{*[31]}$, 产率 $72 \%$. 黄色固体. ${ }^{1} \mathrm{H}$ NMR (400 $\left.\mathrm{MHz}, \mathrm{CD}_{2} \mathrm{Cl}_{2}\right) \delta$ : 10.10 (s, 1H), 8.29 (s, 1H), 7.90 (m, 3H), $7.26(\mathrm{dt}, J=5.6,2.4 \mathrm{~Hz}, 2 \mathrm{H}), 4.17(\mathrm{t}, J=6.1 \mathrm{~Hz}, 2 \mathrm{H}), 3.42$ (t, $J=6.7 \mathrm{~Hz}, 2 \mathrm{H}), 1.76 \sim 2.03(\mathrm{~m}, 4 \mathrm{H})$ (*在小剂量下测 试了含叠氮化合物的熔点, 但在 $90{ }^{\circ} \mathrm{C}$ 以上时飞溅, 未 进行进一步的测试. 下同).

\subsection{3 叠氮化巴比妥酸衍生物 $\mathbf{S 3}$ 的合成}

向预置磁子的 $250 \mathrm{~mL}$ 干燥三颈瓶中, 加入化合物 S2 $(0.8 \mathrm{~g}, 3.0 \mathrm{mmol})$, 巴比妥酸 $(1.2 \mathrm{~g}, 9.3 \mathrm{mmol})$ 和干燥 的乙醇 $(60 \mathrm{~mL})$, 升温至 $80{ }^{\circ} \mathrm{C}$, 回流反应 $10 \mathrm{~h}$. 停止加 热并冷却至室温, 过滤得到橘黄色沉淀物, 随后使用热 乙醇多次洗涤, 得到 $450 \mathrm{mg}$ 叠氮化巴比妥酸衍生物 S3*[26-27], 产率 40\%. 黄色固体. ${ }^{1} \mathrm{H}$ NMR (400 MHz, $d_{6}$-DMSO) $\delta: 11.38(\mathrm{~s}, 1 \mathrm{H}), 11.25(\mathrm{~s}, 1 \mathrm{H}), 8.70(\mathrm{~s}, 1 \mathrm{H})$, $8.41(\mathrm{~s}, 1 \mathrm{H}), 8.30(\mathrm{dd}, J=8.9,1.6 \mathrm{~Hz}, 1 \mathrm{H}), 7.93(\mathrm{~d}, J=9.1$ $\mathrm{Hz}, 1 \mathrm{H}), 7.83$ (d, $J=8.8 \mathrm{~Hz}, 1 \mathrm{H}), 7.41(\mathrm{~d}, J=2.3 \mathrm{~Hz}, 1 \mathrm{H})$, 7.24 (dd, $J=8.9,2.4 \mathrm{~Hz}, 2 \mathrm{H}), 4.18$ (t, $J=6.3 \mathrm{~Hz}, 2 \mathrm{H}), 3.46$ $(\mathrm{d}, J=6.8 \mathrm{~Hz}, 2 \mathrm{H}), 1.76 \sim 2.03(\mathrm{~m}, 4 \mathrm{H})$; HRMS calcd for $\mathrm{C}_{19} \mathrm{H}_{17} \mathrm{~N}_{5} \mathrm{O}_{4}[\mathrm{M}+\mathrm{H}]^{+}:$380.1314, found 380.1327.

\subsection{4 分子刷聚合物 PA-g-PAzo/Bar 的合成}

将 $\mathrm{CuBr}$ (9 mg, $0.058 \mathrm{mmol})$ 和大分子引发剂 poly(Br-acrylate-alkyne) (8 mg, $0.029 \mathrm{mmol}$ ATRP 引发基 团与炔基基团)加入到干燥的预置搅拌子的 Schlenk 瓶 (使用前真空火烤两次) 中, 以橡皮塞封口. 经过三次抽 真空通氮气后加入偶氮苯单体 $(1.9 \mathrm{~g}, 4.36 \mathrm{mmol})$, 化合 物 S3 (56 mg, $0.15 \mathrm{mmol})$, THF (15 mL) 和 DMF (3 mL). 经三次冷冻-抽真空-融解通氮气循环以除去反应液中 的氧气, 加入配体 PMDETA $(15 \mu \mathrm{L}, 0.058 \mathrm{mmol})$ 再进行 一次冷冻-抽真空-融解通氮气循环. 将反应管置于恒温 的 $80{ }^{\circ} \mathrm{C}$ 油浴中加热反应 $6.5 \mathrm{~h}$, 取出用液氮淬灭反应. 粗产物用 $\mathrm{THF}$ 稀释, 过中性氧化铝柱除去 $\mathrm{CuBr}$, 滤液 浓缩后在冰盐浴冷却的正己烷/乙醚 $(V: V=2: 1)$ 中沉 淀, 反复 5 次, 抽真空至恒重得到分子刷聚合物 PA-g-PAzo/Bar (4). ${ }^{1} \mathrm{H}$ NMR (400 MHz, $\mathrm{CD}_{2} \mathrm{Cl}_{2}$ ) $\delta$ : 0.90 $\left(\mathrm{CH}_{2} \mathrm{CH}_{2} \mathrm{CH}_{2} \mathrm{CH}_{3}, \mathrm{CH}_{3} \mathrm{CHCO}_{2}\right), 1.28,1.37,1.62\left(\mathrm{CH}_{2}-\right.$ $\mathrm{CH}_{2} \mathrm{CH}_{2} \mathrm{CH}_{3}, \mathrm{CH}_{2} \mathrm{CHCO}_{2}, \mathrm{CH}_{2} \mathrm{CCO}_{2}, \mathrm{OCH}_{2} \mathrm{CH}_{2} \mathrm{CH}_{2} \mathrm{CH}_{2}-$ $\left.\mathrm{CH}_{2} \mathrm{CH}_{2} \mathrm{O}\right), 2.30\left(\mathrm{CH}_{2} \mathrm{CHCO}_{2}\right), 2.61\left(\mathrm{CH}_{2} \mathrm{CH}_{2} \mathrm{CH}_{2} \mathrm{CH}_{3}\right)$, 3.96, $4.05\left(\mathrm{OCH}_{2} \mathrm{CH}_{2} \mathrm{CH}_{2} \mathrm{CH}_{2} \mathrm{CH}_{2} \mathrm{CH}_{2} \mathrm{O}, \mathrm{CH}_{2} \mathrm{CCO}_{2} \mathrm{CH}_{2}\right.$,
$\left.\mathrm{CH}_{2} \mathrm{CCH}_{2} \mathrm{OCO}\right), 6.92,7.27,7.76\left(\mathrm{C}_{6} \mathrm{H}_{4} \mathrm{~N}_{2} \mathrm{C}_{6} H_{4}\right), 8.27$, 8.43, $10.08\left(\mathrm{C}_{3} \mathrm{~N}_{2} \mathrm{H}_{2} \mathrm{CH}, \mathrm{C}_{10} H_{6}\right)$; FT-IR (KBr) v: 2933, 2855, 1731, 1595, 1570, 1501, 1458, $1253 \mathrm{~cm}^{-1}$; GPC: $M_{\mathrm{n}}=30100, \emptyset=1.27$.

3.2.5 分子刷聚合物胶束溶液的制备与光致异构化 过程

首先将合成的含偶氮苯侧链的分子刷聚合物 PA-g-PAzo/Bar 溶解在良溶剂氯仿中, 充分溶解后过滤. 然后取 $3 \mathrm{~mL}$ 聚合物溶液, 在室温下使用微量注射泵以 流速 $1 \mathrm{~mL} \cdot \mathrm{h}^{-1}$ 向其中加入选择性溶剂甲基环已烷，并低 速摚拌. 待滴加完毕后, 再搅拌 $2 \mathrm{~h}$, 促使聚合物在混合 溶剂中充分组装. 分别配制最终浓度为 0.2 和 $1 \mathrm{mg} \cdot$ $\mathrm{mL}^{-1}$ 的聚合物胶束溶液, 进行紫外光谱以及透射电镜 的测试.

采用强度为 $129 \mathrm{~mW} \cdot \mathrm{cm}^{-2}$ 的 $365 \mathrm{~nm}$ 紫外光源和 $450 \mathrm{~nm}$ 的可见光源, 先后照射分子刷聚合物的胶束溶 液, 胶束溶液的试剂瓶用锡箔纸密封遮光, 并放置在冰 水浴中，保持低速摚拌. 光照不同时间后，分别取样制 备胶束样品, 利用 TEM 观察胶束的形貌转变. 通过紫 外-可见光吸收光谱研究胶束溶液的光致异构化行为时, 直接在仪器样品血中照射不同时间, 实时利用仪器检测 样品紫外吸收峰变化.

辅助材料(Supporting Information) 中间体 S1 S3 的 核磁共振氢谱谱图. 这些材料可以免费从本刊网站 (http://sioc-journal.cn/)上下载.

\section{References}

[1] Cabral, H.; Miyata, K.; Osada, K.; Kataoka, K. Chem. Rev. 2018, $118,6844$.

[2] Mai, Y.; Eisenberg, A. Chem. Soc. Rev. 2012, 41, 5969.

[3] Zhang, K.; Jiang, M.; Chen, D. Prog. Polym. Sci. 2012, 37, 445.

[4] Hayward, R. C.; Pochan, D. J. Macromolecules 2010, 43, 3577.

[5] Tao, D.; Feng, C.; Cui, Y.; Yang, X.; Manners, I.; Winnik, M. A.; Huang, X. J. Am. Chem. Soc. 2017, 139, 7136.

[6] Tao, D.; Feng, C.; Lu, Y.; Cui, Y.; Yang, X.; Manners, I.; Winnik, M. A.; Huang, X. Macromolecules 2018, 51, 2065.

[7] Wu, J.; Liu, Z.; Yao, Y.; Lin, S. Chin. J. Org. Chem. 2019, 39, 2952 (in Chinese). (吴嘉诚, 刘争卉, 姚远, 林绍梁, 有机化学, 2019, 39, 2952.)

[8] Lutz, J.-F.; Lehn, J.-M.; Meijer, E. W.; Matyjaszewski, K. Nat. Rev. Mater. 2016, 1, 16024.

[9] Matyjaszewski, K. Science 2011, 333, 1104.

[10] Polymeropoulos, G.; Zapsas, G.; Ntetsikas, K.; Bilalis, P.; Gnanou, Y.; Hadjichristidis, N. Macromolecules 2017, 50, 1253.

[11] Gregory, A.; Stenzel, M. H. Prog. Polym. Sci. 2012, 37, 38.

[12] Que, Y.; Liu, Y.; Tan, W.; Feng, C.; Shi, P.; Li, Y.; Huang, X. ACS Macro Lett. 2016, 5, 168.

[13] Ju, M.; Xu, B.; Xu, L. Chin. J. Org. Chem. 2020, 40, 4344 (in Chinese). (鞠明杰, 徐涁涁, 徐立功, 有机化学, 2020, 40, 4344.)

[14] Xie, G.; Martinez, M. R.; Olszewski, M.; Sheiko, S. S.; Matyjaszewski, K. Biomacromolecules 2019, $20,27$.

[15] Feng, C.; Huang, X. Acc. Chem. Res. 2018, 51, 2314. 
[16] Xu, B.; Feng, C.; Hu, J.; Shi, P.; Gu, G.; Wang, L.; Huang, X. ACS Appl. Mater. Interfaces 2016, 8, 6685.

[17] Xu, B.; Liu, Y.; Sun, X.; Hu, J.; Shi, P.; Huang, X. ACS Appl. Mater. Interfaces 2017, 9, 16517.

[18] Cai, X.; Hu, W.; Lu, G.; Huang, X. Chin. J. Org. Chem. 2013, 33, 2520 (in Chinese).

(蔡晓冰, 胡薇, 陆国林, 黄晓宇, 有机化学, 2013, 33, 2520.)

[19] Wang, W.; Shen, D.; Li, X.; Yao, Y.; Lin, J.; Wang, A.; Yu, J.; Wang, Z. L.; Hong, S. W.; Lin, Z.; Lin, S. Angew. Chem. Int. Ed. 2018, 57, 2139.

[20] Liu, M.; Liu, H.; Yan, D.; Zhang, Z.; Guan, J. Chin. J. Org. Chem. 2020, 40, 125 (in Chinese).

(刘曼曼, 刘豪浩, 漂道仁, 张智勇, 关金涛, 有机化学, 2020 , 40, 125.)

[21] Cheng, X.; Miao, T.; Yin, L.; Ji, Y.; Li, Y.; Zhang, Z.; Zhang, W.; Zhu. X. Angew. Chem. Int. Ed. 2020, 59, 9669.

[22] Huang, S.; Chen, Y.; Ma, S.; Yu. H. Angew. Chem. Int. Ed. 2018, $57,12524$.

[23] Xu, B.; Qian, H.; Lin, S. ACS Macro Lett. 2020, 9, 404.

[24] Xu, B.; Feng, C.; Huang, X. Nat. Commun. 2017, 8, 333.

[25] Datta, S.; Kato, Y.; Higashiharaguchi, S.; Aratsu, K.; Isobe, A.;
Saito, T.; Prabhu, D. D.; Kitamoto, Y.; Hollamby, M. J.; Smith, A. J.; Dalgliesh, R.; Mahmoudi, N.; Pesce, L.; Perego, C.; Pavan, G. M.; Yagai, S. Nature 2020, 583, 400.

[26] Yagai, S.; Goto, Y.; Lin, X.; Karatsu, T.; Kitamura, A.; Kuzuhara, D.; Yamada, H.; Kikkawa, Y.; Saeki, A.; Seki, S. Angew. Chem. Int. Ed. 2012, 51, 6643.

[27] Adhikari, B.; Yamada, Y.; Yamauchi, M.; Wakita, K.; Lin, X.; Aratsu, K.; Ohba, T.; Karatsu, T.; Hollamby, M. J.; Shimizu, N.; Takagi, H.; Haruki, R.; Adachi, S.; Yagai, S. Nat. Commun. 2017, 8, 15254.

[28] Prabhu, D. D.; Aratsu, K.; Kitamoto, Y.; Ouchi, H.; Ohba, T.; Hollamby, M. J.; Shimizu, N.; Takagi, H.; Haruki, R.; Adachi, S.; Yagai, S. Sci. $A d v$. 2018, 4, eaat8466.

[29] Foster, J. C.; Varlas, S.; Couturaud, B.; Coe, Z.; O’Reilly, R. K. J. Am. Chem. Soc. 2019, 141, 2742-2753.

[30] Manzano, J. I.; Cochet, F.; Boucherle, B.; Gomez-Perez, V.; Boumendjel, A.; Gamarro, F.; Peuchmaur, M. Eur. J. Med. Chem. 2016, $123,161$.

[31] Li, Z.; Zeng, Q.; Li, Z.; Dong, S.; Zhu, Z.; Li, Q.; Ye, C.; Di, C.; Liu, Y.; Qin, J. Macromolecules 2006, 39, 8544. 